\title{
Rhabdomyosarcoma Metastasis to the Breast: Appearance on Diffusion-Weighted Imaging and Apparent Diffusion Coefficient
}

\section{Sequences}

\author{
Chermaine Deepa Antony, ${ }^{1,}{ }^{*}$ Kartini Rahmat, ${ }^{1}$ Anushya Vijayananthan, ${ }^{1}$ and Jayalakshmi Pailoor ${ }^{2}$ \\ ${ }^{1}$ Department of Biomedical Imaging, Faculty of Medicine, University Malaya, Kuala Lumpur, Malaysia \\ ${ }^{2}$ Department of Pathology, University Malaya, Kuala Lumpur, Malaysia \\ "Corresponding author: Chermaine Deepa Antony, Department of Biomedical Imaging, Faculty of Medicine, University Malaya, 50603, Kuala Lumpur, Malaysia. Tel: \\ +60-30379494422, Fax: +60-379492030, E-mail: chermaine.antony@gmail.com
}

Received 2014 September 15; Revised 2015 February 05; Accepted 2015 May 19.

\begin{abstract}
Rhabdomyosarcoma is a common primary childhood malignancy that rarely metastasizes to the breast. We report a patient with a primary sinonasal rhabdomyosarcoma metastasizing to the breast, with no distant spread. We describe the imaging appearance of rhabdomyosarcoma on magnetic resonance imaging (MRI), particularly on diffusion-weighted imaging (DWI) and apparent diffusion coefficient (ADC) sequences. Rhabdomyosarcoma metastasis has a variable appearance on ultrasound and MRI, and may mimic common benign tumors. However, it appears hyperintense on DWI and produces a low value on the ADC, which may be more accurate for determining its malignant nature.
\end{abstract}

Keywords: Rhabdomyosarcoma, Breast, Magnetic Resonance Imaging, Diffusion Magnetic Resonance Imaging

\section{Introduction}

The most common sources of extra-mammary metastases to the breast are melanomas, lymphosarcomas, oat cell tumors of the lung, and ovarian carcinomas (1). Breast metastases from rhabdomyosarcoma are rare, with an incidence varying from $1 \%$ to $5 \%$, occurring mainly in adolescent females. The primary tumor most commonly originates from the extremities. These tumors are also mostly of the alveolar subtype (2). Bilateral involvement is between $8 \%$ and $25 \%$ (1). There are few articles that describe the imaging findings of rhabdomyosarcoma metastases to the breast on magnetic resonance imaging (MRI) (1-3). The variable morphological and enhancement patterns of benign and metastatic lesions to the breast on MRI suggest limited specificity of standard conventional contrastenhanced MRI in differentiating malignant from benign lesions (4-6). Recent studies have demonstrated the evolving role of diffusion-weighted imaging (DWI) and proton spectroscopy in the characterization of breast lesions (7-9).

We report a rare case of breast metastasis from a sinonasal rhabdomyosarcoma occurring in a 13-year-old girl, in particular describing the imaging features with an emphasis on DWI and apparent diffusion coefficient(ADC), which current reports have noted to be useful sequences in discriminating between benign and malignant breast lesions.

\section{Case Presentation}

A 13-year-old girl presented to the surgical department with a rapidly growing, painless mass in her left breast over the previous one month. On physical examination, there was a large $(10 \mathrm{~cm})$, round, hard, immobile, mildly tender mass located in the outer portion of the left breast. No axillary lymph nodes were palpable.

One year earlier, the patient had been found to have embryonal rhabdomyosarcoma of the left maxillary sinus with local extension into the left ethmoid and frontal sinuses, associated with ipsilateral cervical lymphadenopathy. The patient underwent gross total resection of the tumor after four cycles of neoadjuvant chemotherapy. The margins of the resected tumor were not clear. Subsequently, she completed another five cycles of chemotherapy along with radiotherapy. The initial metastatic workup, which included a CT of the chest, bone scintigraphy and bone marrow biopsy, was negative. Blood tests were unremarkable.

During this presentation, she underwent another computed tomography (CT) of the chest, which showed an ill-defined mass in the left breast with ipsilateral axillary lymphadenopathy (Figure $1 \mathrm{~A}$ and $\mathrm{B}$ ). Breast ultrasound was performed using a $12.5 \mathrm{MHz}$ linear array transducer (Phillips IU22 Philips Healthcare, Bothell, WA, USA). This revealed a large ill-defined heterogeneous lesion containing necrotic areas and demonstrating increased vascular- 
ity (Figure 1C and D).

Subsequently, a biopsy of the mass was performed and revealed a rhabdomyosarcoma, which was cytologically identical to the primary lesion. The microscopic sections showed strips of breast tissue infiltrated by large nests of viable malignant small round blue cells. These cells were mildly pleomorphic, and demonstrated hyperchromatic nuclei with minimal cytoplasm. Numerous mitoses were seen with aberrant forms. The surrounding stroma was desmoplastic, with interspersed infiltrating strands and cords of these malignant cells and myogenin, consistent with tumor of rhabdomyoblastic origin (Figure 2).

After the biopsy, the patient underwent another two cycles of chemotherapy. Due to her young age and the aggressive nature of the disease, head and breast MRI was used for surveillance and assessment of the treatment response. The examination was performed on a 3.0 Tesla MRI machine with a dedicated bipolar phased-array breast coil (GE Healthcare, Milwaukee, WI, USA). The pre-contrast series (T2-weighted and STIR) were acquired before the injection of the contrast agent. Axial T1-weighted 3D spoiled gradient-recalled echo pulse sequences were performed with the following characteristics: echo time $=2.1 \mathrm{~ms}$, repetition time $=4.3 \mathrm{~ms}$, flip angle $=10^{\circ}, \mathrm{FOV}=36 \mathrm{~cm}$, slice thickness $1.4 \mathrm{~mm}$, and matrix size $=256 \times 256$ pixels. The dynamic contrast-enhanced images were sequentially obtained before, and six phases after, the administration of a $10 \mathrm{ml}$ bolus of intravenous gadopentetate dimeglumine at $0,60,120,180,240,300$, and 360 seconds $(0.2$ $\mathrm{mmol} / \mathrm{kg}$ of Gd-DTPA followed by $20 \mathrm{ml}$ of normal saline). Breast subtraction dynamic contrast-enhanced MRI (BS DCE-MRI) scanning, performed before chemotherapy and two months later, showed marked shrinkage of the tumor. On pre-chemotherapy MRI, the lesion appeared ill-defined, with heterogeneous signal intensity on STIR images, and demonstrating heterogeneous enhancement on BS DCEMRI. It measured approximately $4.5(\mathrm{AP}) \times 2.8(\mathrm{~W}) \times 4.5$ (H) $\mathrm{cm}$ (Figure $3 \mathrm{~A}-\mathrm{E}$ ). On the dynamic images, there was rapid early enhancement with the arterial peak at 180-240 seconds after contrast medium injection, followed by flattening during the delayed phase (type II kinetic curve)(Figure $3 \mathrm{~F}$ ). There was also infiltration of the underlying pectoralis muscle. Follow-up BS DCE-MRI post-chemotherapy demonstrated multiple small and well-defined peripheral enhancing residual lesions, indicating a good treatment response (Figure $3 \mathrm{G}$ ). The breast lesion demonstrated restricted diffusion on DWI and a low ADC value of $0.25 \times 10$ $-3 \mathrm{~mm}^{2} / \mathrm{s}$.

\section{Discussion}

There are three articles in the literature that report the MR appearance of rhabdomyosarcoma metastasis to the breast. Birjawi et al. reported a case of an 11-year-old girl with breast metastases from a primary sinonasal tumor, which was described as showing an intermediate signal on T1-weighted images, heterogeneous hyperintensity on T2-weighted images, hyperintensity on STIR, and diffuse enhancement with central necrosis (3). Perlet et al. described breast metastases from rhabdomyosarcoma as well-circumscribed nodular lesions with early, rapid ringlike enhancement, with the largest lesion displaying very low signal intensity centrally, indicating necrosis (2). Wurdinger et al. also reported a similar early, rapid ringenhancement followed by subsequent confluent enhancement (1). Interestingly, the tumor in our case displayed heterogeneous enhancement. Therefore, it is not always possible to differentiate malignant versus benign lesions on MR imaging, and the differential diagnosis in this case would include benign breast lesions, such as fibroadenomas, as well as lymphomas and metastases from other primary sites.

In cases of metastases, MRI is useful in affirming their malignant nature, as they tend to exhibit a washout phenomenon identical to that of primary carcinomas. However, the specificity of conventional MRI in detecting malignancy is limited, as some benign lesions have features that are indistinguishable from cancers, including rapid contrast enhancement (4-6). In this case, the histologically proven rhabdomyosarcoma metastatic tumor demonstrated a type II curve with relatively rapid contrast uptake up to 180 seconds after contrast medium injection, which may be seen in some malignant lesions.

Sen et al. (10) reported the accuracy of differentiating benign from malignant lesions based on size, morphology, signal intensity on T1- and T2-weighted imaging, and postgadolinium appearance. Margin irregularity was the most specific indicator of malignancy, with an accuracy of $78 \%$. The rest of the features described were only able to differentiate benign from malignant masses with an accuracy of $58 \%$ to $78 \%$ (11). Findings of benign tumors, primary breast carcinomas, or metastases may overlap on MRI, and histological tissue confirmation is usually warranted in patients with a known primary malignancy (4). In this respect, DWI and ADC sequences may substantiate findings of malignancy in lesions that exhibit a morphology and an enhancement kinetic curve that are suspicious for malignancy.

The ADC of a tissue is expressed in units of $\mathrm{mm} 2 / \mathrm{s}$, with no unanimity regarding the boundaries of the range of normal diffusion, but ADC values less than 1.0 to $1.1 \times 10^{-3}$ 

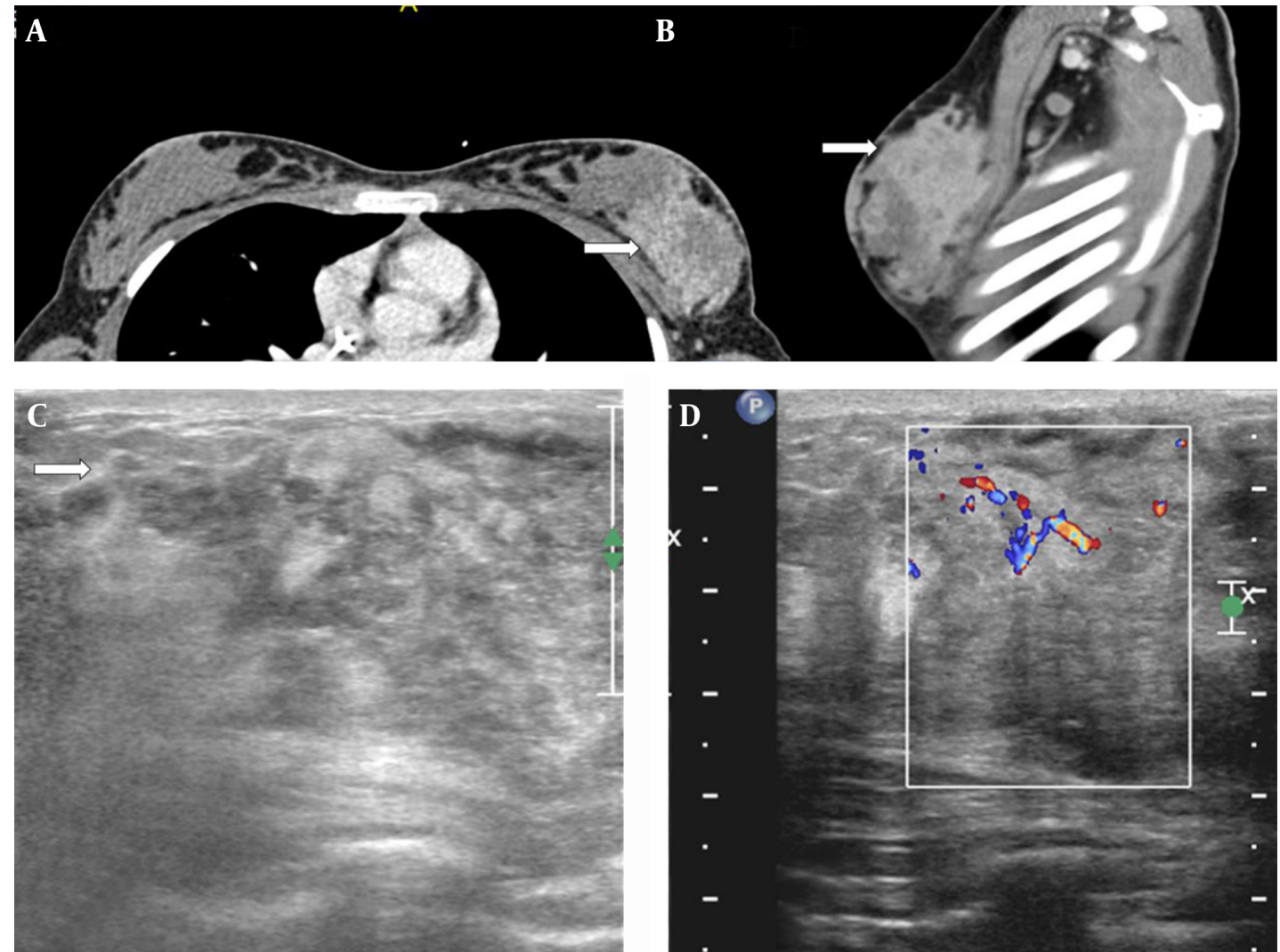

Figure 1. A 13-year-old girl with history of embryonal rhabdomyosarcoma of the maxillary sinus presented with a rapidly growing painless mass in left breast. A, Contrastenhanced CT of the chest (white arrow) shows a large heterogeneously enhancing mass occupying the outer half of the left breast on axial (white arrow) and B, sagittal (white arrow) images; C, Ultrasound of the left breast shows a large heterogeneous mass with poorly defined margins (white arrow); D, Color Doppler demonstrates increased peripheral vascularity.
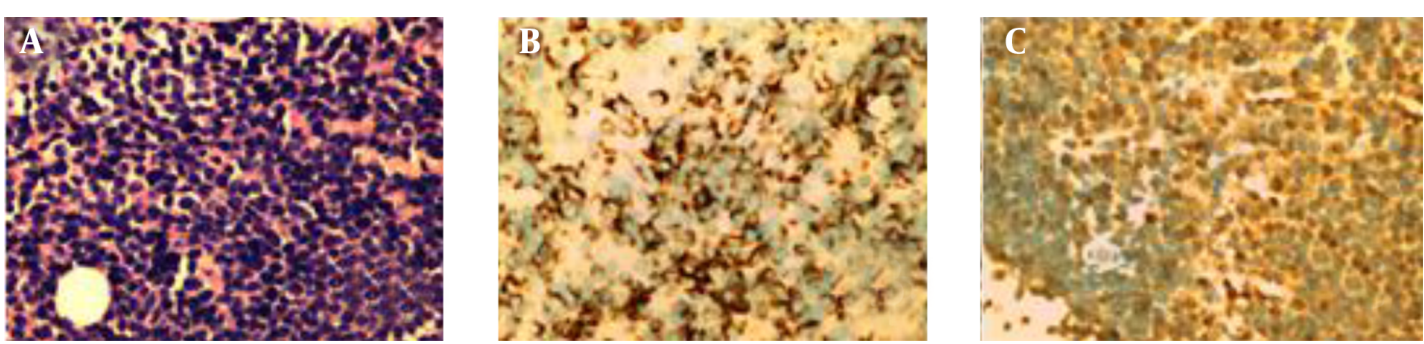

Figure 2. A, Sheets of round tumor cells with hyperchromatic nuclei and scanty cytoplasm. Lack of alveolar pattern. (Hematoxylin \& Eosin staining, magnification $\times 200$ ); B, Tumor cells positive for desmin. (Immunostain for desmin, magnification $\times 200$ ); C, Tumor cells showing nuclear immunopositivity. (Immunostain for myogenin, magnification $\times 200)$

$\mathrm{mm}^{2} / \mathrm{s}$ generally indicate restriction in diffusion (11). However, a study comparing the normal breast parenchymal ADC values with mammographic density, age, and background parenchymal enhancement revealed a strong correlation between mammographic density and ADC values, with ADC values increasing with breast density (7).

Tan and colleagues found that the ADC values of malig- nant lesions were significantly lower than those of benign lesions, with the cut-off ADC value for malignant lesions being $1.22 \times 10^{-3} \mathrm{~mm}^{2} / \mathrm{s}$ for $\mathrm{b}=1000 \mathrm{~s} / \mathrm{mm}$ (8). Our study gave a low ADC value of $0.25 \times 10^{-3} \mathrm{~mm}^{2} / \mathrm{s}$, consistent with a malignant lesion, which is consistent with Tan et al.'s report (8). Our case demonstrates the usefulness of the ADC value as a marker for breast malignancy, especially in young pa- 

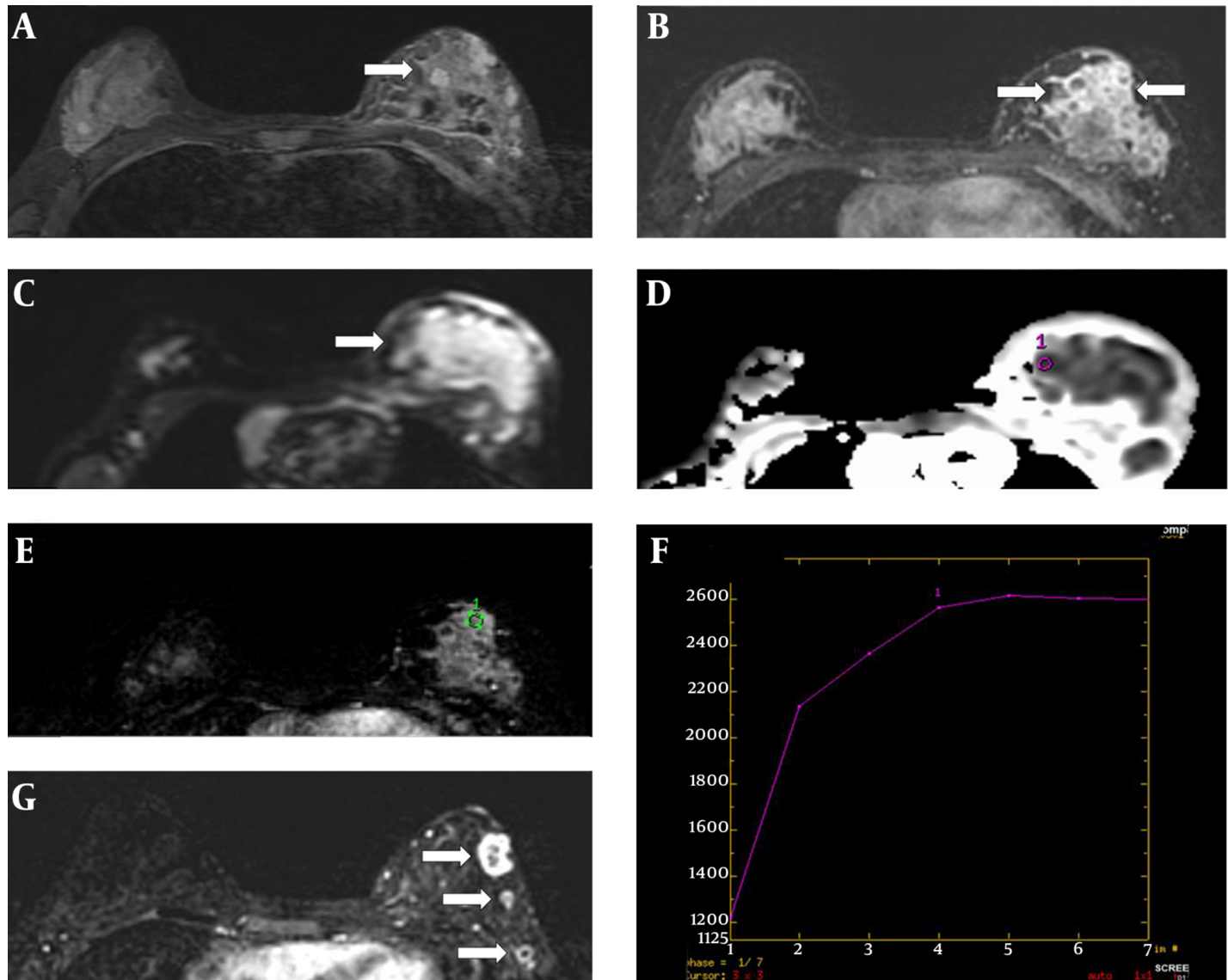

Figure 3. Breast MRI. A, Axial STIR image showing a heterogeneous mass (white arrow); B, Axial image of early subtraction dynamic MR showing an ill-defined heterogeneously enhancing mass (white arrows); C, Axial DWI MRI showing hyperintense signal (white arrow); D, Axial section of the ADC map showing an ROI cursor (purple circle) placed over a hypointense area within the tumor (black arrow); the area calculated for the tumor had an ADC value of $0.25 \times 10-3 \mathrm{~mm} 2 / \mathrm{s} \mathrm{at} \mathrm{a} \mathrm{b-value} \mathrm{of} 1000 \mathrm{~mm} 2 / \mathrm{s}$; E, Dynamic curve with ROI (green circle) placed over the enhancing solid tissue; F, Dynamic curve shows a plateau (type II) curve. The curve reaches a peak during the arterial phase 180-240 seconds after contrast medium injection, followed by a flattening during the delayed phase; G, Axial image of early subtraction dynamic MR post-chemotherapy showed significantly reduced tumor size, with multiple well-circumscribed hypointense lesions demonstrating rim enhancement (white arrows).

tients with dense breasts.

A recent report by Bahreini et al. (12) in 2010 showed that the gradient vector flow (GVF) snake segmentation method and BS DCE-MRI give more diagnostic information about breast lesions, with a reported sensitivity of $81 \%$ for both methods and a specificity of $60 \%$ when the GVF snake segmentation method is used on the first series alone. They concluded that their method, if used correctly in addition to the DWI and ADC sequences, may provide better diagnostic information (3).

Previous reports of rhabdomyosarcoma metastases to the breast $(1,2,3,13)$ demonstrated that a poor prognosis despite treatment, and relapse of this aggressive tumour, was observed in most patients (Table 1). Early diagnosis is recommended when suspicious lesions are detected in patients with primary rhabdomyosarcoma. Although immunocytochemical studies are definitive (14) and may be necessary in some cases; breast MRI with diffusionweighted imaging is a useful non-invasive diagnostic tool that may help avoid the need for biopsy in cases with a high clinical suspicion of breast metastasis.

\section{Acknowledgments}

The authors gratefully acknowledge the technical support from the UM research imaging centre (UMRIC), and would like to thank Prof. Dr. Wan Ariffin, Prof. Dr. Nur Aishah, and Dr. Nazri Md. Shah for their contributions to this case report.

\section{Footnotes}

Authors' Contribution: CDA, KR and AV drafted the manuscript. CDA, KR, AV and JP edited the contents and ap- 
Table 1. Clinical Data of Various Reports in the Literature

\begin{tabular}{|c|c|c|c|c|c|}
\hline & Histology & Primary & Secondary & Treatment & Outcome \\
\hline Wurdinger et al.(1) & Alveolar type & Bladder & $\begin{array}{l}\text { Lymph node, bone } \\
\text { marrow, Both } \\
\text { breasts }\end{array}$ & $\begin{array}{l}\text { High-dose chemotherapy with } \\
\text { autogenous hematopoietic stem cell } \\
\text { transplantation, Relapse with bilateral } \\
\text { breast involvement }\end{array}$ & $N A^{a}$ \\
\hline Perlet et al.(2) & $\begin{array}{l}\text { Poorly differentiated } \\
\text { alveolar type }\end{array}$ & Left upper extremity & Both breasts & $\begin{array}{l}\text { Four cycles of chemotherapy combined } \\
\text { with local hyperthermia, followed by } \\
\text { resection and local radiation therapy of } \\
\text { primary tumor, Relapse with bilateral } \\
\text { breast involvement }\end{array}$ & $N A^{\mathrm{a}}$ \\
\hline Birjawi et al.(3) & $\begin{array}{l}\text { Embryonal or solid } \\
\text { area of alveolar type }\end{array}$ & $\begin{array}{l}\text { Nasal cavity and } \\
\text { ethmoid sinus }\end{array}$ & Right breast & $\begin{array}{l}\text { Nine cycles of chemotherapy and } \\
\text { radiotherapy, with primary tumor } \\
\text { decreasing in size, Relapse with bilateral } \\
\text { breast lesions, followed by recurrence of } \\
\text { tumor at the primary site and metastatic } \\
\text { pleural effusions }\end{array}$ & $N A^{a}$ \\
\hline \multicolumn{6}{|l|}{ Chateil et al.(13) } \\
\hline Case 1 & Alveolar type & Unknown & Breast & Chemotherapy Regression of tumors & $N A^{a}$ \\
\hline Case 2 & Rhabdomyosarcoma & Right elbow & Breast & $\begin{array}{l}\text { Chemotherapy Remission, Recurrence } \\
\text { with bone marrow extension }\end{array}$ & Death \\
\hline Case 3 & Alveolar type & Pelvic mass & Breast & $\begin{array}{l}\text { Chemotherapy, Recurrence several } \\
\text { months later }\end{array}$ & Death \\
\hline Case 4 & Rhabdomyosarcoma & $\begin{array}{l}\text { Right maxillary } \\
\text { sinus }\end{array}$ & Right breast & $\begin{array}{l}\text { Chemotherapy, Relapse with skeletal } \\
\text { extension }\end{array}$ & Death \\
\hline
\end{tabular}

${ }^{\mathrm{a}}$ Not available

proved final version for submission.

Funding/Support: Dr. Kartini Rahmat received funding from these institutional grants: RP008B-13HTM and RG39011HTM.

\section{References}

1. Wurdinger S, Schutz K, Fuchs D, Kaiser WA. Two cases of metastases to the breast on MR mammography. Eur Radiol. 2001;11(5):802-6. doi: 10.1007/s003300000636. [PubMed: 11372611].

2. Perlet C, Sittek H, Forstpointner R, Kessler M, Reiser M. Metastases to the breast from rhabdomyosarcoma: appearances on MRI. Eur Radiol. 1999;9(6):1113-6. doi: 10.1007/s003300050801. [PubMed:10415245].

3. Birjawi GA, Haddad MC, Tawil AN, Khoury NJ. Metastatic rhabdomyosarcoma to the breast. Eur Radiol. 2001;11(4):555-8. [PubMed: 11354746].

4. Iglesias A, Arias M, Santiago P, Rodriguez M, Manas J, Saborido C. Benign breast lesions that simulate malignancy: magnetic resonance imaging with radiologic-pathologic correlation. Curr Probl Diagn Radiol. 2007;36(2):66-82. doi: 10.1067/j.cpradiol.2006.12.001. [PubMed: 17331838].

5. Kaiser WA. False-positive results in dynamic MR mammography. Causes, frequency, and methods to avoid. Magn Reson Imaging Clin N Am. 1994;2(4):539-55. [PubMed: 7489307].

6. Orel SG, Schnall MD, LiVolsi VA, Troupin RH. Suspicious breast lesions: MR imaging with radiologic-pathologic correlation. Radiology. 1994;190(2):485-93. doi: 10.1148/radiology.190.2.8284404. [PubMed: $8284404]$.
7. McDonald ES, Schopp JG, Peacock S, DeMartini WB, Rahbar H, Lehman CD, et al. Diffusion-weighted MRI: association between patient characteristics and apparent diffusion coefficients of normal breast fibroglandular tissue at 3 T. AJR Am J Roentgenol. 2014;202(5):W496-502. doi: 10.2214/AJR.13.11159. [PubMed: 24758685].

8. Tan SL, Rahmat K, Rozalli FI, Mohd-Shah MN, Aziz YF, Yip CH, et al. Differentiation between benign and malignant breast lesions using quantitative diffusion-weighted sequence on 3 T MRI. Clin Radiol. 2014;69(1):63-71. doi:10.1016/j.crad.2013.08.007. [PubMed: 24156797].

9. Suppiah S, Rahmat K, Mohd-Shah MN, Azlan CA, Tan LK, Aziz YFA, et al. Improved diagnostic accuracy in differentiating malignant and benign lesions using single-voxel proton MRS of the breast at 3 T MRI. Clinical Radiology. 2013;68(9):e502-10. doi: 10.1016/j.crad.2013.04.002. [PubMed: 23706826].

10. Sen J, Agarwal S, Singh S, Sen R, Goel S. Benign vs malignant soft tissue neoplasms: limitations of magnetic resonance imaging. Indian J Cancer. 2010;47(3):280-6. doi: 10.4103/0019-509X.64725. [PubMed: 20587903].

11. Haaga JR. CT and MRI of the Whole Body. Mosby/Elsevier; 2008.

12. Bahreini L, Fatemizadeh E, Guity M. Diagnostic Efficacy of All Series of Dynamic Contrast Enhanced Breast MR Images Using Gradient Vector Flow (GVF) Segmentation and Novel Border Feature Extraction for Differentiation Between Malignant. Iran J Radiol. 2010;7(4):225-34.

13. Chateil JF, Arboucalot F, Perel Y, Brun M, Boisserie-Lacroix M, Diard F. Breast metastases in adolescent girls: US findings. Pediatr Radiol. 1998;28(11):832-5. doi: 10.1007/s002470050476. [PubMed: 9799312].

14. Owji S, Kumar P, Noorani H. Breast metastatic alveolar rhabdomyosarcoma: FNA findings. Iran Red Crescent Med J. 2008;10(1):38-40. 